\title{
Merging of Native and Non-native Speech for Low-resource Accented ASR
}

\author{
Sarah Samson $\operatorname{Juan}^{1(\bowtie)}$, Laurent Besacier ${ }^{2}$, Benjamin Lecouteux ${ }^{2}$, \\ and Tien-Ping $\operatorname{Tan}^{3}$ \\ 1 Faculty of Computer Science and Information Technology, \\ Universiti Malaysia Sarawak, Kota Samarahan, Sarawak, Malaysia \\ sjsflora@unimas.my \\ 2 Grenoble Informatics Laboratory (LIG), \\ University Grenoble-Alpes, Grenoble, France \\ \{laurent. besacier, benjamin. lecouteux\}@imag.fr \\ ${ }^{3}$ School of Computer Science, Universiti Sains Malaysia, Gelugor, Penang, Malaysia \\ tienping@cs.usm.my
}

\begin{abstract}
This paper presents our recent study on low-resource automatic speech recognition (ASR) system with accented speech. We propose multi-accent Subspace Gaussian Mixture Models (SGMM) and accent-specific Deep Neural Networks (DNN) for improving non-native ASR performance. In the SGMM framework, we present an original language weighting strategy to merge the globally shared parameters of two models based on native and non-native speech respectively. In the DNN framework, a native deep neural net is fine-tuned to non-native speech. Over the non-native baseline, we achieved relative improvement of $15 \%$ for multi-accent SGMM and $34 \%$ for accent-specific DNN with speaker adaptation.
\end{abstract}

Keywords: Automatic speech recognition - Cross-lingual acoustic modelling $\cdot$ Non-native speech $\cdot$ Low-resource system $\cdot$ Multi-accent SGMM • Accent-specific DNN

\section{Introduction}

Performance of non-native automatic speech recognition (ASR) is poor when few (or no) non-native speech is available for training / adaptation. Many approaches have been suggested for handling accented-speech in ASR, such as acoustic model merging $[2,16,22,23]$, applying maximum likelihood linear regression (MLLR) for adapting models to each non-native speaker [8], or adapting lexicon $[1,4]$.

Lately, Subspace Gaussian Mixture Models (SGMMs) $[17,18]$ have shown to be very promising for ASR in limited training conditions (see $[11,13]$ ). In SGMM modelling, the acoustic units are all derived from a common GMM called the Universal Background Model (UBM). This UBM, which in some way represents the acoustic space of the training data, can be estimated on large amount of 